\title{
TRANSVERSE IMPEDANCE MEASUREMENTS OF THE DARHT-2 ACCELERATOR CELL*
}

\author{
R. Briggs, SAIC, Pleasanton, CA; D. Birx, SRL (deceased), S. Nelson, LLNL, Livermore, CA \\ L. Reginato and M. Vella, LBNL, Berkeley, CA
}

\begin{abstract}
A new technique was developed to measure the transverse interaction impedance of the DARHT-2 induction cells, designed to accelerate a 2-4 kA, $2 \mu \mathrm{sec}$ electron beam pulse. An extensive campaign to minimize the transverse resistive impedance of these massive metglas-filled structures lead to a design with thin ferrite tiles in the form of a ring placed along one wall of the radial line connecting the pulseline feeds to the accelerator gap. This ferrite ring heavily damped all transverse modes. To measure the broadband $m=1$ impedance, one cell is excited by a matched twinlead on the axis of the beamtube. The ratio of the radial RF magnetic field in the accelerator gap to the azimuthal RF magnetic field at the beamtube wall (far away from the gap) determines the complex impedance, as explained in the paper. This technique is much simpler than the TSD approach for these very large bore systems. It also covers the full frequency band including the reactive impedance at low frequency related to the "image displacement instability". Results with the 10 inch diameter bore "standard cell" indicate a peak resistive impedance of $280 \mathrm{ohms}$ per meter around $575 \mathrm{MHz}(\mathrm{Q}=6)$.
\end{abstract}

\section{INTRODUCTION}

In designing the DARHT-2 accelerator cells, minimization of the transverse impedance controlling beam breakup instability (BBU) growth has been a very high priority for meeting the project objectives.[1] The requirement for minimal transverse displacements (excellent emittance) is driven by the small spot size needed for radiography, and the 2 microsecond pulselength at beam currents up to $4 \mathrm{kA}$ makes high $\mathrm{Q}$ modes especially dangerous. The high vacuum requirements, driven by the long pulselength and utilization of thermionic cathodes, made it desirable if not mandatory to use ceramic insulators with their high dielectric constant. Impedance mismatches in the gap region were therefore a major concern initially.

A campaign combining theoretical modeling and impedance measurements was mounted to optimize the cell design for minimal BBU; details of this overall campaign will be published separately. In this paper, we describe a new approach that was developed to meet the challenge of measuring the broadband transverse impedance of these massive, heavily damped cells. Techniques like TSD and frequency shifts for Z/Q determination were used in initial studies, but found to be

* This work was performed under the auspices of the U.S. Dept. of Energy by LBNL under contract AC03-76SF00098 very difficult and/or inadequate for a final determination of the impedance, especially in the 14" diameter bore injector cells. The existence of modes as low as $50 \mathrm{MHz}$, for example, illustrates the nature of the difficulties.

In this paper, we focus mainly on the new technique for measuring the transverse impedance that was developed. Highlights of the results obtained with the final cell configuration are given in the last section.

\section{DARHT-2 ACCELERATOR CELL}

A cutaway view of the DARHT-2 standard cell containing its four metglas cores is shown in Figure 1. Arrow \#1 points to the ferrite tiles put in to damp the BBU modes, in the form of a ring placed on the back plate of the radial line that feeds power to the accelerator gap. Arrow \#2 points to the high dielectric constant mycalex insulator that separates the vacuum beamline region from the oil insulation region containing the metglas.

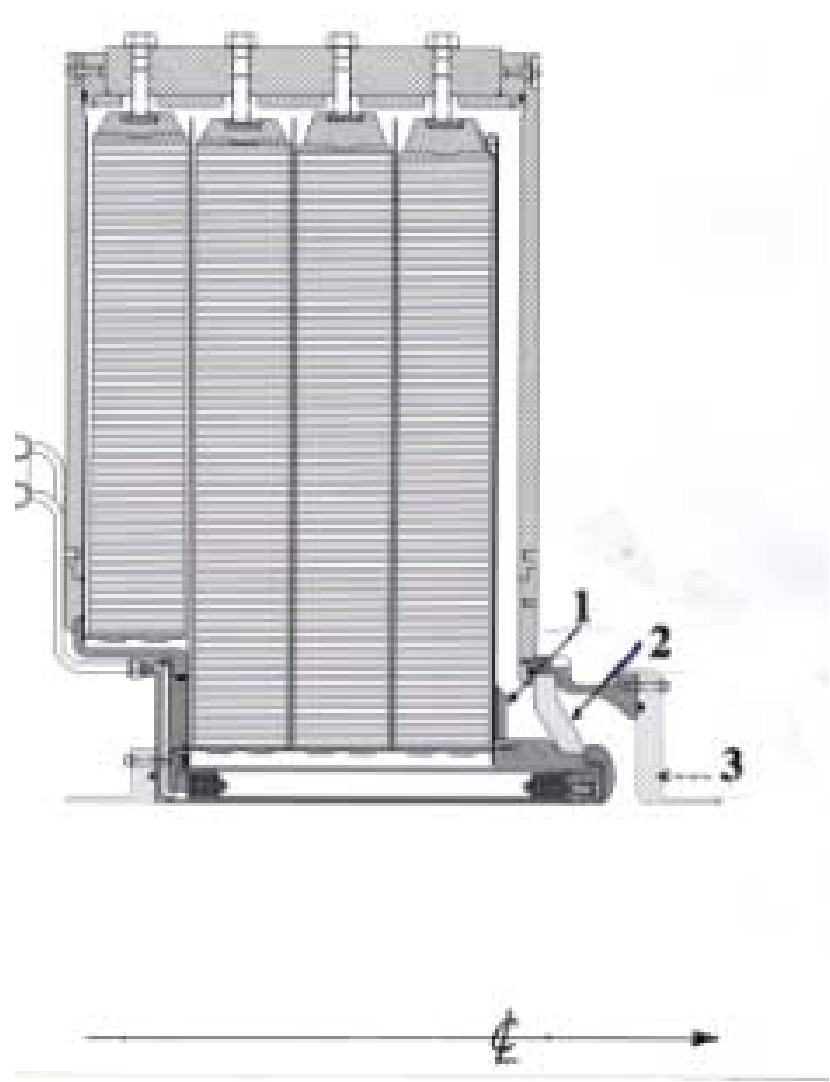

Figure 1: A cutaway view of the DARHT-2 standard cell 


\section{MEASUREMENT TECHNIQUE}

The Basic Idea: A twin lead is placed along the axis of the accelerator cell with several foot long beam tube sections connected on both sides. A signal generator connected to the twin lead excites dipolar surface currents on the beam tube wall in the form of a traveling wave since the twin lead is terminated in a matched resistive load. The impedance measurement technique consists of measuring the phase and amplitude of the radial dipole if magnetic field just inside the accelerator gap, $B_{r g}$, and the azimuthal dipole rf magnetic field at the beam tube wall, ${ }^{B} \theta w$, at an axial position removed by several beam tube radii from the gap. We define the ratio of these two complex amplitudes, a dimensionless number not requiring any loop calibrations, as

$$
\eta=B_{r g} / B_{\theta w}
$$

where the phase differences between the two measurements is adjusted to account for the propagation of the TEM wave on the twin lead from the measurement position of $B_{\theta w}$ to the gap midplane. The transverse interaction impedance is then given by

$$
Z_{\perp}=-j \sqrt{\frac{\mu_{O}}{\varepsilon_{O}}} \frac{w}{\pi b^{2}} \eta \text { ohms/meter }
$$

where $w$ is the gap width and $b$ is the beam tube radius. The BBU gain is directly proportional to the real (resistive) part of $Z_{\perp}$, and the peak value of the dimensionless parameter $\operatorname{Im}(\eta)$ should be comparable to unity in a well-damped cell.

Theoretical Basis of the Measurement Technique: A relativistic electron beam of current I oscillating transversely with amplitude $\Delta_{x}$ in the $\mathrm{x}$ direction inside a conducting beam tube of radius $\mathrm{b}$ induces a dipole surface current on the tube wall equal to

$$
K_{z}=-\frac{I \Delta_{x}}{\pi b^{2}} \cos \theta=K_{z o} \cos \theta
$$

The beam also induces a dipole surface charge $K_{z} / c$ on the tube wall; the collective forces from the surface charge (attractive) and the surface current (repulsive) cancel inside a smooth conducting tube for a relativistic beam, as is well known. When the beam passes through an accelerating gap region, the dipole surface current flows out into the accelerator cell, destroying this force balance. We consider only frequencies below the cutoff for propagation in the beam tube $\left(\omega<\omega_{c o}=1.84 c / b\right)$ where the dipole modes of the cavity excited by the beam oscillation lead to localized deflection forces on the beam.

To determine the net transverse impulse imparted to the electrons passing through the gap region, we need to integrate the transverse electromagnetic force $E+v \times B$ along their trajectory in $\mathrm{z}$ (assumed to be at a constant displacement from the axis through the gap region). For highly relativistic electrons, it can be proven that this integral is independent of radial position anywhere inside the beam tube. Using this theorem, we can relate the change in transverse momentum to the radial magnetic field at the gap:

$$
\delta p_{x}=-e \int B_{r}(r=b, \theta=\pi / 2) d z
$$

This radial magnetic field is of course directly proportional to the strength of the current source $K_{z o}$ (and it varies as $\sin \theta$ ); the properties of the cell itself can therefore be incorporated into a dimensionless (frequency dependent) parameter defined by

$$
\eta=-\frac{1}{w} \int B_{r g} d z / \mu_{0} K_{z 0}=<B_{r g}>/ B_{\theta w}
$$

where $B_{r g}$ is shorthand notation for the radial field at $r=b$ and $\theta=\pi / 2$ in Eq. 4 , and $<>$ implies an average over the gap. Introducing the definition of transverse interaction impedance

$$
\delta p_{x}=-j \frac{e}{c} Z_{\perp} I \Delta_{x}
$$

into Eq. 4, and using Equations 3 and 5, we obtain Eq. 2 (with $\eta$ now defined by the more precise formula of Eq. 5). Note that the measurement of $B_{\theta}$ at the wall some distance axially from the accelerator gap $(z>3 b$ in the experiments) provides a direct measure of the "source current" $K_{z o}$ consistent with Eq. 5.

This discussion carries over directly to the measurement of the transverse impedance with a twin lead excitation. The role of the twin lead is to induce a surface current in the beam tube of the same form as Eq. 3, except that $\Delta_{x}$ is now the spacing of the wires and $\pm I$ is the current in each wire. To simulate the beam, the wave launched on the twin lead should be a pure forward (or backward) traveling wave, that is, the reflections from the interaction with the gap should be minimal (achieved by close spacing of the wires).

Correction factors for practical features of rounded gaps and finite loop size: The actual gap has rounded corners as shown in Fig.1. The loop measuring $B_{r}$ in the gap was mounted at the radius indicated by arrow \#3 in Fig.1, defined by $r_{p}=b+\Delta r$, where $\Delta r=5 / 4 "$ was the position where the gap was a minimum $(w=1 ")$, and the loop extended axially across almost all the gap. A correction factor for this can be developed by integrating $\operatorname{div} B=0$ over $\mathrm{z}$ at a fixed $\mathrm{r}$ inside the gap to obtain

$$
\frac{\partial}{\partial r}\left(r<B_{r}>\right)=<B_{\theta}>
$$

where the dependence $\sin \theta$ for $B_{r}$ and $\cos \theta$ for $B_{\theta}$ is implicit. By performing an additional measurement, namely the azimuthal dipole field in the gap at the same radius as $B_{r g}$ (same loop mounting hole, rotated by 90 
degrees in theta), the $\mathrm{z}$ integral of the radial field can be linearly interpolated inward to the tube radius. Define the (negative) ratio of this measured azimuthal gap field to the radial gap field as

$$
s=-<B_{\theta g}\left(r_{p}\right)>/<B_{r g}\left(r_{p}\right)>
$$

Let $\eta_{p}$ be the value measured by the loop at $r_{p}$. Then the "true $\eta$ " can be obtained from Eq. 7 and Eq. 8 as

$$
\eta=\eta_{p}\left[1+\frac{\Delta r}{b}(1+s)\right]
$$

An additional (smaller) correction arises from the finite extent of the loop inward from the wall (by $\delta=3 / 4 "$ ) in the measurement of $B_{\theta w}$; this is accounted for by multiplying the RHS of Eq. 9 by an additional factor $c_{\theta}=(1-\delta / 2 b) /(1-\delta / b)$.

\section{RESULTS}

Measurements on a standard cell were made using a network analyzer driving a 61" long twinlead made from $0.5^{\prime \prime}$ copper rods spaced by $1.5^{\prime \prime}$ and terminated in a 210 ohm resistor (the twinlead characteristic impedance). The network analyzer was connected to the twinlead through a balun, and ferrite blocks were placed around the twinlead at the source end, to provide common mode discrimination (an issue requiring due diligence in these measurements). In addition, the rf loops were actually a matched set of two loops on opposite sides of the beamtube (cell) connected in an "up-up" orientation to select the dipole field components.

The measured complex $\eta$ (eta) for the standard cell is presented in Fig. 2 (the blue line). The cutoff frequency for the 10" diameter bore standard cell is $692 \mathrm{MHz}$, so data above about $650 \mathrm{MHz}$ are not meaningful. The transverse impedance is $189 \operatorname{Im}($ eta) for these cell dimensions. Note that eta approaches a real (negative) value around unity at low frequencies. This quasi-static force tending to pull the beam towards the wall is the origin of the well known "image displacement instability" in induction linacs.

It is standard practice to approximate the transverse interaction impedance by a set of discrete resonant modes, in the form

$$
\eta=\sum_{n} \frac{j \eta_{M n}}{1+j 2 Q_{n}\left(\omega-\omega_{0 n}\right) / \omega_{0 n}}+\eta_{0}
$$

For each resonant frequency, there are two free parameters to adjust to get the best fit to the data: the peak value of $\operatorname{Im} \eta$ at resonance (related to the usual Z/Q parameter), and the width of the resonance $(\mathrm{Q})$. After a few iterations, necessary because of the overlap of these low $Q$ resonances, the values in Table 1 were chosen to obtain a reasonable match between the model and the $\operatorname{Im} \eta$ vs. frequency data. (Note: we found the slope of the $\operatorname{Re} \eta$ vs. frequency at resonance to also be useful for making initial estimates of $\mathrm{Q}$ in these iterations). Once these parameters are chosen to fit the resistive part of the impedance, the reactive component of the impedance is then completely determined (except for a real constant). A value of $\eta_{0}=-0.7$ was chosen in the equation above to make the low frequency value of $\operatorname{Re} \eta$ match the data).
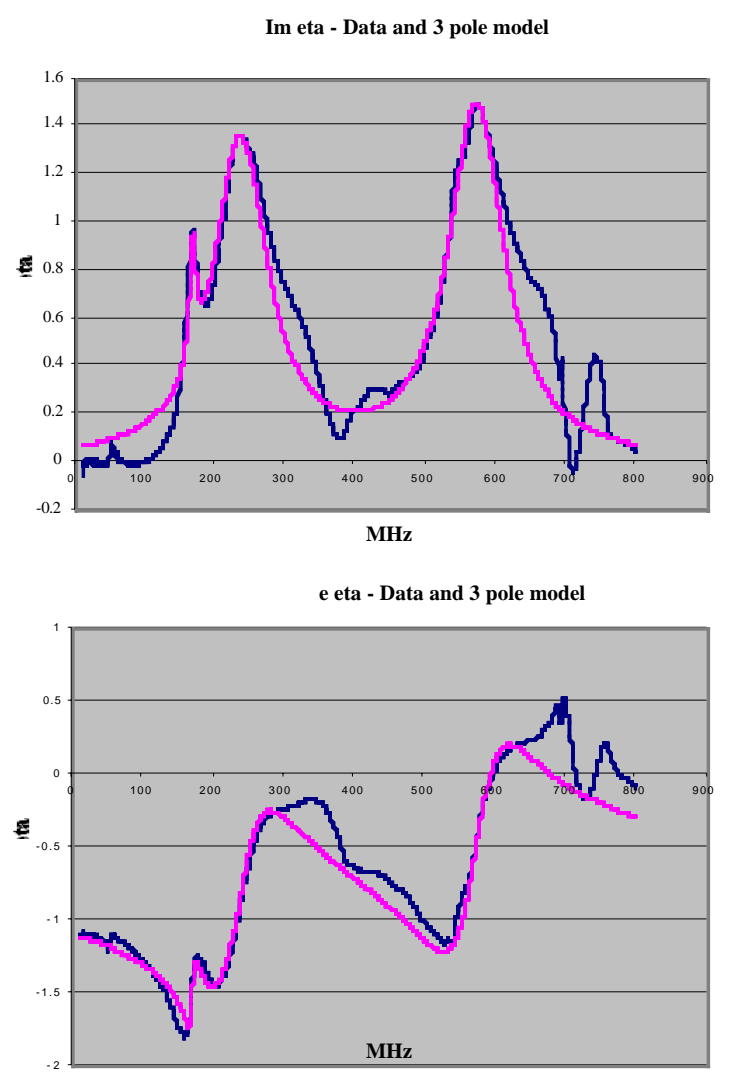

Fig. 2: Data for DARHT-2 standard cell (blue line) and a resonant mode model (red line).

Table 1: Resonant mode parameters

\begin{tabular}{|l|l|l|}
\hline$f_{n}$ (exp. peak) & $\eta_{M n}$ & $Q_{n}$ \\
\hline $168.5 \mathrm{MHz}$ & 0.49 & 15 \\
\hline $236 \mathrm{MHz}$ & 1.33 & 2.5 \\
\hline $572 \mathrm{MHz}$ & 1.47 & 5.88 \\
\hline
\end{tabular}

These parameters and a similar set for the injector cells were used to calculate BBU for typical DARHT-2 tunes. [2]

\section{REFERENCES}

[1] M. Burns, "Status of the DARHT Phase 2 Long-Pulse Accelerator", paper WOAA008, this conference.

[2] Y. Chen and W. Fawley, "BBU and Corkscrew Growth Predictions for the DARHT Second Axis Accelerator", paper RPPH108, this conference. 